\title{
Efectos del entrenamiento cardiovagal en la respuesta autonómica en personas con sobrepeso Effects of cardiovagal training on autonomic response in overweight people \\ *.**Alexis Espinoza-Salinas, **José González-Jurado, ***Arnaldo Burdiles-Álvarez, *Giovanny Arenas-Sánchez, *Marcelo Bobadilla-Olivares \\ *Universidad Santo Tomás (Chile), **Universidad Pablo de Olavide (España), ***Universidad San Sebastián (Chile)
}

Resumen. La asociación entre la obesidad y un mayor riesgo de morbimortalidad cardiometabólica es un hecho, pero las alteraciones metabólicas provocan cambios autonómicos que no siempre son detectados en una institución de salud. Objetivo: Evidenciar el efecto de un protocolo interválico de alta intensidad sobre la actividad cardiovagal en personas con sobrepeso, evaluando por medio de la variabilidad de la frecuencia cardiaca. Método: Colaboraron 20 personas con sobrepeso, sin otros antecedentes mórbidos. Su edad fue de

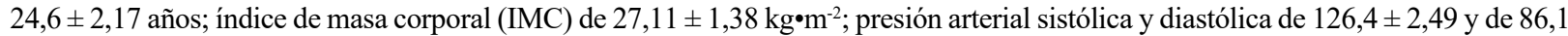
$\pm 1,85 \mathrm{mmHg}$, respectivamente. El muestreo fue realizado por conveniencia. Se distribuyeron aleatoriamente en Grupo de intervención (GI, $\mathrm{n}=10$ ) y Grupo de Control (GC, $\mathrm{n}=10)$. Resultados: Se registraron diferencias significativas entre los grupos, en los tiempos donde los valores de inicio son menores que a la sexta semana (F: 120,412; G.L. $=1 ; 7 ; \mathrm{p}=0,003)$. Además, existe diferencia estadística entre el valor SD1 del GC > GE (F: 6,700; G.L. $=1 ; 7 ; p=0,036)$ y solamente en el GI, entre la 1 semana $>6$ semana (EMMEANS $=10,400$; $\mathrm{p}=0,001)$. Conclusión: En seis semanas de intervención, con un protocolo de activación vagal, aumentó la modulación parasimpática en reposo en personas con sobrepeso.

Palabras clave: Obesidad, síndrome metabólico, variabilidad de la frecuencia cardiaca, modulación autonómica, ejercicio de alta intensidad.

Summary. The association between obesity and an increased risk of cardiometabolic morbidity and mortality is a fact, but most of the time there are metabolic disorders that cause autonomic changes not always detected by health institutions. Objective: To demonstrate the effect of a high intensity and short duration interval protocol on cardiovagal activity in overweight people through the evaluation of heart rate variability. Method: a total of 20 overweight people without other morbid backgrounds collaborated in the study. Their age was $24.6 \pm 2.17$ years old; body mass index (BMI) of $27.11 \pm 1.38 \mathrm{~kg} \bullet \mathrm{m}-2$; systolic and diastolic blood pressure of $126.4 \pm 2.49$ and 86.1 $\pm 1.85 \mathrm{mmHg}$, respectively. Sampling was done by convenience. They were randomly distributed in Intervention Group (GI, $\mathrm{n}=10)$ and Control Group (GC, $n=10)$. Results: Significant differences were registered between the groups, the starting values being lower than after sixth week (F: 120,412; G.L. $=1 ; 7 ; \mathrm{p}=0,003)$. In addition, there is a statistical difference between the SD1 value of the GC $>\mathrm{GE}$ (F: 6,700; GL $=1 ; 7 ; \mathrm{p}=0.036$ ) and only in the GI, between 1 week $>6$ weeks (EMMEANS $=10,400 ; \mathrm{p}=0.001$ ). Conclusion: After the six-week intervention with a vagal activation protocol, parasympathetic modulation at rest increased in overweight people.

Keywords: Obesity, metabolic syndrome, heart rate variability, autonomic modulation, high intensity exercise.

\section{Introducción}

La obesidad es un trastorno metabólico de origen multifactorial. Su incidencia ha ido en aumento, tanto en países desarrollados como Estados Unidos y en vías de desarrollo, y ha llevado a un incremento de comorbilidades asociadas, principalmente diabetes tipo 2 y enfermedades cardiovasculares (Cuadri-Fernández, 2017;Torres, 2015; Hall, 2014).

En ella, el incremento de tejido adiposo se asocia a una mayor liberación de adipocitoquinas. Un ejemplo de esto son la leptina, interleuquinas y adiponectina, que pueden mediar cambios metabólicos como: la hipertensión arterial, la resistencia a la insulina (RI) y dislipidemia. En esta línea, la obesidad ha sido asociada con alteraciones en la función del sistema nervioso autónomo (SNA), debido a una alteración del balance autonómico sobre el nódulo sinusal (Sekine, 2001; Marques, Pastre, Freitas \& Fernandes, 2010). Un incremento del tejido adiposo se ha relacionado con una mayor descarga simpática en condiciones de reposo (Álvarez, Stacy, Beske, Ballar \& Kevyn, 2002), además de tener una menor actividad vagal vinculada a una disminución de la sensibilidad barorrefleja (Farah, Wagner, Thiago \& Raphael, 2013; Skrapari etal., 2007).

Fecha recepción: 28-08-19. Fecha de aceptación: 04-12-19 Alexis Espinoza-Salinas

alespinozasalinas@gmail.com
Adicionalmente, se ha establecido que en la población obesa existe una fuerte asociación con la RI y un estado de hiperinsulinemia, generando una alteración en el balance autonómico del corazón (Windham, 2012; Straznicky, 2014), estableciendo, así, una disminución de la sensibilidad del nódulo sinusal a las influencias del SNA. (Kuller et al., 1991).

Uno de los métodos para valorar la función autonómica es la variabilidad de la frecuencia cardiaca (VFC) (Stein et al., 2009), la cual engloba el análisis de las variaciones de los intervalos entre ondas R sucesivas en un electrocardiograma (Kingsley \& Figueroa, 2014). La VFC no solo aporta información acerca de la regulación electrofisiológica y autonómica, sino también de las fluctuaciones respiratorias, respuestas barorreflejas y modificaciones circadianas que determinan la frecuencia cardiaca (Force,1996). Existen diferentes métodos de valoración de la VFC, entre los cuales encontramos los métodos lineales, no lineales y espectrales (Poddar et al, 2015; Stein et al, 2009; Lewis \& McNarry, 2013). Los métodos lineales entregan información de los dominios de tiempo y frecuencia del VFC (Poddar et al., 2015). Los no lineales nos informan acerca del comportamiento fractal de la VFC (aleatoriedad de los patrones de frecuencia entre las series de tiempo de los intervalos R-R) y del balance simpático vagal. Por último, los métodos espectrales cuantifican la cantidad de variación en los intervalos R-R a diferentes frecuencias, entregando información de la actividad del SNA, pero también del sistema renina angiotensina aldosterona y 
del patrón respiratorio (Stein et al., 2009). Se ha observado que las personas obesas presentan mayores alteraciones en la VFC, con una modulación simpática aumentada y paralelamente una disminución de la actividad vagal (Skrapari et al., 2007).

El ejercicio de forma regular está asociado con adaptaciones positivas en la composición corporal, condición física, niveles de inflamación crónica e índices de VFC favorables, especialmente reflejados en un aumento de la modulación vagal y una reducción de la activación del Sistema Nerviosos Simpático (SNS) (Straznicky et al., 2014). Esta modificación del balance autonómico, que favorece la activación del Sistema Nervioso Parasimpático (SNP), se ha asociado a una inhibición de la inflamación crónica por descarga simpática, suprimiendo la síntesis y liberación de citoquinas pro inflamatorias(Windham et al., 2012). Debido al mayor riesgo que se presenta en esta población en desarrollar anormalidades en la VFC y de padecer eventos cardiacos, es que ha sido muy difícil establecer la dosificación de ejercicio físico en este grupo etario (Straznicky et al., 2014).

Históricamente, los programas de ejercicio en modalidad aeróbica continua y últimamente de fuerza, han sido utilizados como los principales métodos de intervención no invasiva para mejorar los parámetros del VFC y disminuir el riesgo de padecer una Enfermedad Cardiovascular (ECV). Además, la combinación de ambos protocolos de ejercicio es la vía más efectiva para alcanzar resultados beneficiosos (Windham et al., 2012).

En las últimas décadas ha emergido una nueva estrategia de ejercicio, denominado entrenamiento interválico de alta intensidad (EIAI), que ha demostrado ser eficaz en la mejora del estado físico asociado a la salud de las personas (Gist et al., 2013). Este método consiste en cortas, pero intensas series de ejercicio intercalados, con breves periodos de pausa activa o pasiva (Guiraud et al., 2012; Dupont et al., 2003). La principal ventaja del EIAI es el corto periodo de tiempo que se necesita para completar el entrenamiento, requiriendo un mínimo de equipamiento y adaptaciones físicas. El EIAI produce iguales o mayores ganancias cardiometabólicas en el corto plazo, en comparación al ejercicio aeróbico continúo (Costigan et al., 2015), presentando una percepción del esfuerzo menor, menores niveles de catecolaminas plasmáticas y aumentos en las concentraciones de lactato en sangre (Meyer et al., 1996). Se ha demostrado, además, que el EIAI reduce el riesgo de padecer una ECV en adultos sanos (Lee et al., 2003). Incluso, sorprendentemente, con protocolos de una sola sesión de EIAI a la semana, fue suficiente para reducir el riesgo de muerte por enfermedades cardiovasculares en hombres y mujeres, comparado con sujetos sedentarios (Wisløff et al., 2006; Duarte et al., 2013).

Este estudio busca dejar evidencia del efecto de un protocolo interválico de alta intensidad en la actividad cardiovagal en personas con sobrepeso.

\section{Materiales y método}

\section{Diseño}

Este trabajo presenta un diseño de tipo cuasi experimental, longitudinal, correlacional-causal.

\section{Participantes}

Formaron parte de la muestra 20 personas con sobrepeso, sin otros antecedentes mórbidos. La edad de los participantes fue de 24,6 $\pm 2,17$ años; índice de masa corporal (IMC) de $27,11 \pm 1,38 \mathrm{~kg} \bullet \mathrm{m}^{-2}$; presión arterial sistólica de 126,4 $\pm 2,49$ $\mathrm{mmHg}$ y presión arterial diastólica de $86,1 \pm 1,85 \mathrm{mmHg}$. El muestreo fue realizado por conveniencia. Los individuos participaban en un programa de ejercicio y salud, pertenecientes al Centro de Ejercicio Adaptado (CEA), YMCA, Santiago, Chile. El trabajo consistió en la realización de ejercicio aeróbico continuo, flexibilidad, coordinación, fuerza y estabilización lumbo pélvica, supervisado por educadores físicos, kinesiólogos y médicos. Todos los participantes son físicamente activos ( $>150$ minutos de actividad física de intensidad moderada o vigorosa por semana). Los criterios de exclusión fueron: presión arterial (PA) sobre 130/85 mmHg no controlada y/o alguna patología músculo esquelética, metabólica o sistémica agudas o crónicas, que les impidieran realizar el protocolo de ejercicios o que modificaran los valores de la VFC. Se consideraron las normas de la Declaración de Helsinki para intervención con seres humanos. Todos los voluntarios firmaron el consentimiento informado. Los procedimientos fueron aprobados por el Comité de Ética de la Universidad Santo Tomás, Santiago de Chile.

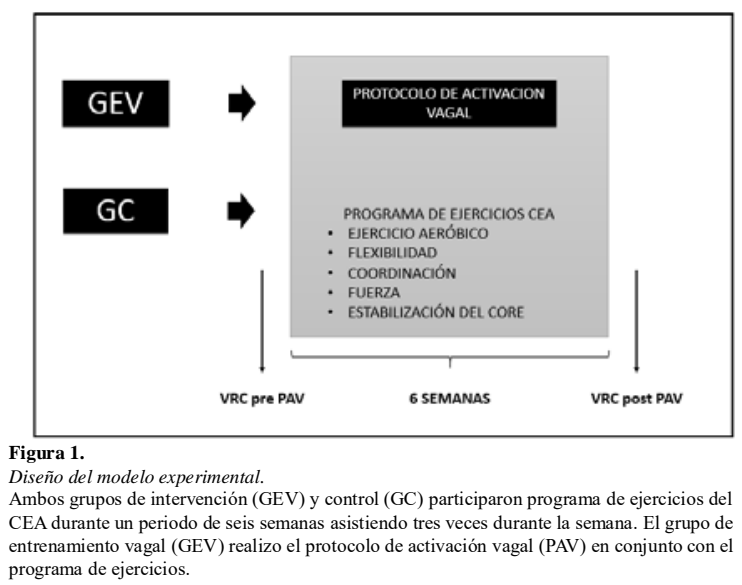

\section{Procedimiento}

En el Laboratorio de Fisiología de la Universidad Santo Tomás, Santiago de Chile, se desarrolló el estudio, en conjunto con el CEA. Las 20 personas fueron distribuidas en dos grupos al azar, grupo de intervención $(\mathrm{GI})(\mathrm{n}=10)$ y grupo control $(\mathrm{GC})(\mathrm{n}=10)$. Los dos grupos se mantuvieron en el programa de ejercicios del CEA durante seis semanas, con una frecuencia de tres veces a la semana. El GI, además del protocolo de base, llevó a cabo el programa de activación vagal. Durante la mañana (entre las ocho y 10 horas), se realizó la medición de la VFC y PA, dos días antes y después de las seis semanas que duró la intervención, (figura 1). Se controló la correcta hidratación de los participantes y se evitó el consumo de alimentos en las dos horas anteriores a la medición. Se valoró la frecuencia cardiaca (FC), PA, y percepción de esfuerzo, por medio de la escala Borg, con fines de seguridad y control, considerando que un valor sobre 15 se interrumpe el protocolo.

\section{Función autonómica}

Antes de la medición de la VFC, los participantes debían 
estar durante 15 minutos en la posición decúbito supino. Para su implementación, se hizo uso de un cardiotacómetro (POLAR modelo RS800CX, Finlandia), que registra la variación de los intervalos R-R en el tiempo. Los intervalos R$\mathrm{R}$ fueron medidos durante un periodo de cinco minutos. A través del software POLAR PROTRAINER 5, se registraron y convirtieron los datos a intervalos en milisegundos (ms) en formato TXT. Estos últimos fueron analizados por el software Kubios HRV® Analysis V2.0 (Universidad de Kuopio, Finlandia), con las respectivas autorizaciones, determinando las características no lineales (SD1) y espectrales (LF, HF y la relación $\mathrm{LF} / \mathrm{HF}$ ) de la $\mathrm{VFC}$.

\section{Variables Hemodinámicas}

Previo a comenzar el protocolo, se determinaron la PA máxima y la FC. Después de 20 minutos de reposo en posición sentada, la PA se midió a ciegas, mediante auscultación, por un especialista, utilizando el equipo Omrom m6 / confort, mientras que la FC se evaluó con un oxímetro de pulso (Saturometro Heal Force ${ }^{\circledR}$ Prince 100F). El doble producto se calculó como FC (lpm) x presión sistólica (PAS) (mmHg). PAS y FC se midieron al mismo tiempo, en dos oportunidades, con intervalos de tres minutos.

\section{Protocolo de activación vagal}

Protocolo de Activación Vagal (PAV) lo definimos como un EIAI de transición ejercicio-reposo de alta intensidad, cuyo objetivo es modificar el balance autonómico, aumentando la activación vagal y favoreciendo una retirada simpática. Desde el punto de vista operacional, nos referimos específicamente al protocolo de Duarte y cols. 2013. Este protocolo se ejecuta sobre un cicloergómetro sin resistencia externa (Monark, modelo Ergomedic 874E), ajustado ergonómicamente a cada individuo, quienes recibieron previamente instrucciones de la ejecución del protocolo. Consta de dos fases: Una, el individuo permanece sentado durante 55 segundos sin pedalear (fase pasiva); Dos, pedaleo a máxima intensidad durante cinco segundos (fase activa). El individuo repite este ciclo cinco veces, con una duración total de cinco minutos. Durante la fase activa, el individuo es alentado a realizarlo a la máxima intensidad. La prueba se realizó en un ambiente indoor, en las instalaciones del CEA.

\section{Análisis estadístico}

En cuanto a las variables cuantitativas, se emplearon la media y la desviación estándar como estadígrafos de descripción. En este estudio, se empleó un análisis de varianza de un factor como medidas repetidas. Los tratamientos o factores en esta prueba fueron grupo control y grupo experimental, y como medidas repetidas se emplearon dos evaluaciones en el tiempo (una y seis semanas). Para estimar las

\begin{tabular}{|c|c|c|c|c|}
\hline \multirow{2}{*}{ Variables } & \multicolumn{2}{|c|}{ GC } & \multicolumn{2}{|c|}{ GI } \\
\hline & Pre PAV & Post PAV & Pre PAV & Post PAV \\
\hline Peso (kg) & $74,5(5,31)$ & $75(3,26)$ & $81,2(3,87)$ & $81(3,83)$ \\
\hline $\mathrm{FC}(\mathrm{lpm})$ & $76,25(3,83)$ & $74,25(2,62)$ & $75,8(4,26)$ & $70,8(4,26)$ \\
\hline Doble Producto & $95,9(7,3)$ & $91,1(6,1)$ & $94,9(7)$ & $88,1(7,4)$ \\
\hline PAS (mmHg) & $124(2,42)$ & $119(1,78)$ & $120(4,35)$ & $115(2,57)$ \\
\hline PAD (mmHg) & $83(3,46)$ & $82(5,02)$ & $79(2,18)$ & $80(3,53)$ \\
\hline $\mathrm{LF}\left(\mathrm{ms}^{2}\right)$ & $2524(5,71)$ & $2431(3,87)$ & $2176(5,17)$ & $1986(3,21)$ \\
\hline $\mathrm{HF}\left(\mathrm{ms}^{2}\right)$ & $1179(6,33)$ & $1197(7,09)$ & $993(6,43)$ & $1154(4,73)$ \\
\hline $\mathrm{LF} / \mathrm{HF}$ & $2,14(0,25)$ & $2,03(0,19)$ & $2,19(0,22)$ & $1,72(0,16)^{*}$ \\
\hline $\mathrm{SD} 1(\mathrm{~ms})$ & $39,8(2,9)$ & $40,7(1,2)$ & $38,6(2,8)$ & $59(7,32)^{*}$ \\
\hline
\end{tabular}

diferencias, se usó EMMEANS, que muestra las medias marginales estimadas de la variable dependiente y sus errores estándar de las medias para los factores especificados. Como significancia estadística, se empleó un valor $\mathrm{p}<.05$. Por último, todas las pruebas estadísticas se realizaron con el programa SPSS V20 (IBM 2011).

\section{Resultados}

\section{Variables hemodinámicas y antropométricas}

La información recopilada de los 20 participantes se presenta en la Tabla 1. No se observaron diferencias en el peso corporal, FC de reposo, doble producto y PA, entre los GC y GI previo a la evaluación (Tabla 1). Posterior a la intervención, se observó una tendencia a disminuir de la FC de reposo en el GI, desde 75,8 $\pm 4,26$ a 70,8 $\pm 4,26$ latidos por minuto. Adicionalmente, en este grupo se mostró una marcada tendencia a disminuir el doble producto $(94,9 \pm 7$ a $88,1 \pm 7,7)$ y la PAS $(120 \pm 4,35$ a $115 \pm 2,57 \mathrm{mmHg})$. Ambos grupos no presentaron cambios en el peso corporal posterior a la intervención.

\section{Variabilidad de la frecuencia cardiaca}

Los resultados muestran que no hay diferencias significativas entre el GI y GC. Diferencias significativas existen en los tiempos donde los valores de inicio son menores que a la sexta semana (F: 16,$565 ;$ G.L. $=1 ; 7 ; p=.005)$ y solo existe diferencia estadística entre el valor LF/HF del GI, entre la semana uno $>$ semana seis $($ EMMEANS $=.474 ; p=.002)$, figura 2. El valor eta-cuadrado parcial calculado para las evaluaciones (Tiempo) fue .703. Considerado como un tamaño del efecto fuerte (Bakeman et al., 2005).

En el caso de la SD1, los resultados muestran que existen diferencias significativas entre el GI y GC. Diferencias significativas existen en los tiempos donde los valores de inicio son menores que a la sexta semana (F: 120,412; G.L. $=1 ; 7 ; p=$ $.003)$. Asimismo, hay diferencia estadística entre el valor SD1 del GC $>$ GI (F: 6,700; GL. $=1 ; 7 ; p=.036)$ y solamente en el GI, entre la semana uno $>$ semana seis $($ EMMEANS $=10,400 ; p=$ $.001)$, figura 3 . El valor eta-cuadrado parcial calculado para las evaluaciones (Tiempo) fue .745. Considerado como un tamaño del efecto fuerte (Bakeman et al., 2005).

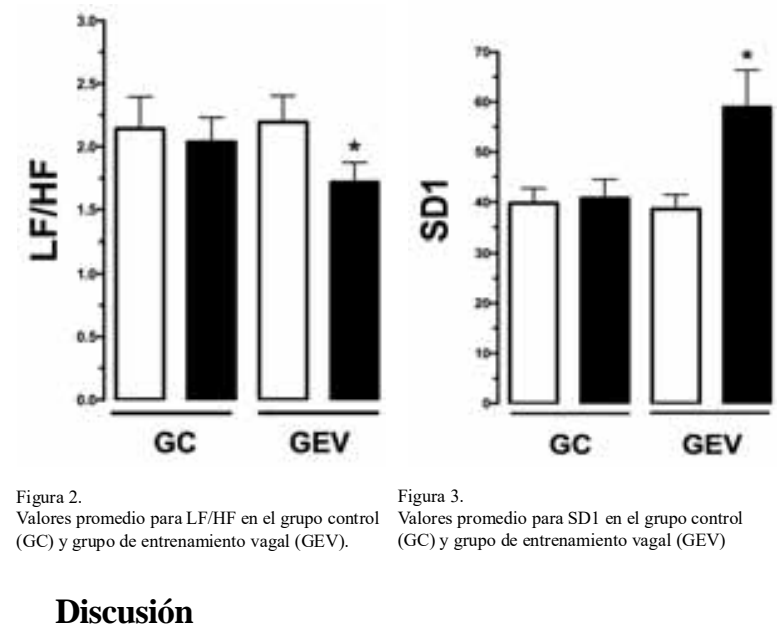

En este trabajo de investigación se analizó la función 
autonómica, a partir del comportamiento electrofisiológico del corazón, frente a la adaptación que implica un ejercicio de transición rápida reposo-ejercicio de alta intensidad. Específicamente, en una modalidad que da énfasis en la activación del SNP. Los resultados muestran que, tras seis semanas de intervención con un PAV, aumentó la modulación vagal de reposo en personas con sobrepeso. Lo anterior, a través de un protocolo de bajo costo y alto beneficio cardiovascular, comparado con otros tipos de estrategias, como es el caso del ejercicio aeróbico continuo.

Duarte et al. no definen el motivo ni los argumentos que respalden la articulación de tiempos e intensidades del protocolo de que utilizamos como modelo. Sin embargo, en este estudio, el protocolo de entrenamiento tuvo gran impacto en las variables cardiovasculares evaluadas. Los dos valores implicados en el balance autonómico mejoraron sustancialmente, tanto a nivel de la distribución de los patrones frecuenciales (valores espectrales), como en el comportamiento multifractal de la VFC (valores no lineares). A pesar de que ambos grupos pertenecían a un programa regular de ejercicio físico, solo el entrenamiento vagal mostró cambios en los parámetros de VFC al término de la intervención. Al parecer el mecanismo fisiológico que responde a las modificaciones cardiovagales, corresponde en un inicio a una disminución inmediata del tono vagal, incrementando el gasto cardiaco y FC durante los primeros cinco segundos. El cese del ejercicio produce sobrecarga de volumen/presión en los cuerpo carotideos y activación del barorreflejo. Con un período de entrenamiento de múltiples repeticiones, la reeducacion del arco reflejo vuelva a la activación vagal inducida por el ejercicio. Inmediatamente finalizado el protocolo de ejercicio se observa una cinética de la FC con un comportamiento bifásico(Wisløff et al., 2006; Gladwell et al., 2010; Marques et al., 2015), caracterizándose por una rápida disminución debido a un incremento de la actividad vagal, seguido de una segunda fase más prolongada en la disminución de la FC en el tiempo por una lenta retirada simpático-adrenal. El PAV repite constantemente esta serie de eventos fisiológicos, generando cambios cíclicos del balance del SNA. La recuperación de la FC está modulada por el balance autonómico y dependerá de los diferentes sistemas que controlen la modulación de la respuesta del SNA posterior al ejercicio, entre ellos esta los mecanismos centrales, el metabolorreflejo y el barorreflejo (Wisløff et al., 2006; Gladwell et al., 2010). Esto nos indica, fuertemente, que la incorporación de un PAV son útiles para lograr adaptaciones cardiovasculares óptimas.

La relevancia de la función que realiza el SNP está en la fase inicial de recuperación post ejercicio, donde existe una rápida disminución de la $\mathrm{FC}$ seguida de una fase más lenta de recuperación. Este comportamiento no solo se debe a un aumento de la actividad vagal, sino que también a una depresión del SNS, mostrando un comportamiento exponencial (Duarte et al., 2013). La literatura reporta que la fase rápida estaría comandada por el sistema nervioso central y la fase lenta por el clearence de metabolitos y señales neurohumorales que modularían el balance autonómico (Marques et al.,2015).

Diferentes tipos de entrenamientos generarán diferentes patrones de recuperación de la $\mathrm{FC}$ y por lo tanto una predominancia variable de las dos ramas del SNA(Wisløff et al., 2006). La predominancia vagal posterior a la realización de este tipo de entrenamiento podría explicarse en parte por las características del patrón de recuperación de la FC generado, aunque son necesarios nuevos estudios que correlacionen estas variables.

Por último, la alta prevalencia de la obesidad y sobrepeso, sumado a los costos económicos asociados, hacen necesario la incorporación de nuevas estrategias para el control y mejora de esta enfermedad. El PAV es una herramienta terapéutica que implica una mayor accesibilidad, menor tiempo de entrenamiento y requiere de un equipamiento de bajo costo, generando mayor adherencia a un programa de entrenamiento.

Como conclusión, los resultados sugieren que sumar un protocolo específico de activación vagal a un programa de entrenamiento multiparamétrico, en sujetos con sobrepeso, mejora sustancialmente la modulación autonómica evaluada por VFC, al compararla con el programa multiparamétrico sin componente cardiovagal. Sin embargo, es necesario una mayor exploración en el área, de tal forma de poder entender los mecanismos fisiológicos implicados y su compatibilidad y extrapolación a otras poblaciones.

\section{Referencias}

Ahmadian, M., Roshan, V. D., \& Hosseinzadeh, M. (2015). Parasympathetic reactivation in children: influence of two various modes of exercise. Clinical Autonomic Research, 25(4), 207-212.

Alvarez, G. E., Beske, S. D., Ballard, T. P., \& Davy, K. P. (2002). Sympathetic neural activation in visceral obesity. Circulation, 106(20), 2533-2536.

Bakeman, R. (2005). Recommended effect size statistics for repeated measures designs. Behav Res Methods. 37(3):379-84.

Cuadri Fernández, J., Tornero Quiñones, I., Sierra Robles, Á., \& Sáez Padilla, J. (2017). Revisión sistemática sobre los estudios de intervención de actividad física para el tratamiento de la obesidad (Systematic Review of Physical Activity Programs for the treatment of Obesity). Retos, O(33), 261-266.

Cunha, F. A., Midgley, A. W., Gonçalves, T., Soares, P. P., \& Farinatti, P. (2015). Parasympathetic reactivation after maximal CPET depends on exercise modality and resting vagal activity in healthy men. SpringerPlus, 4(1), 100.

Droguett, V. S. L., da Cruz Santos, A., de Medeiros, C. E., Marques, D. P., do Nascimento, L. S., \& do Socorro Brasileiro-Santos, M. (2015). Cardiac autonomic modulation in healthy elderly after different intensities of dynamic exercise. Clinical interventions in aging, 10, 203.

Duarte, C., de Castro, C., \& de Araujo, C. (2013). Treinamento para disfunção vagal cardíaca com repetições da transição repouso-exercício. Revista Brasileira de Atividade Física \& Saúde, 18(6), 688-688.

Dupont, G., Blondel, N., \& Berthoin, S. (2003). Performance for short intermittent runs: active recovery vs. passive recovery. European journal of applied physiology, 89(6), 548-554.

Farah, B. Q., Prado, W. L. D., Tenório, T. R. D. S., \& Ritti-Dias, 
R. M. (2013). Heart rate variability and its relationship with central and general obesity in obese normotensive adolescents. Einstein (Sao Paulo), 11(3), 285-290.

Force, T. (1996). Standards of measurement, physiological interpretation and clinical use. Task force of the European Society of Cardiology and the North American Society of Pacing and Electrophysiology. Circulation, 93(5), 1043-1065.

Gist, N. H., Fedewa, M. V., Dishman, R. K., \& Cureton, K. J. (2014). Sprint interval training effects on aerobic capacity: a systematic review and meta-analysis. Sports medicine, 44(2), 269-279.

Gladwell, V. F., Sandercock, G. R. H., \& Birch, S. L. (2010). Cardiac vagal activity following three intensities of exercise in humans. Clinical Physiology and Functional Imaging, 30(1), 17-22.

Guiraud, T., Nigam, A., Gremeaux, V., Meyer, P., Juneau, M., \& Bosquet, L. (2012). High-intensity interval training in cardiac rehabilitation. Sports medicine, 42(7), 587-605.

Hall, M. E., do Carmo, J. M., da Silva, A. A., Juncos, L. A., Wang, Z., \& Hall, J. E. (2014). Obesity, hypertension, and chronic kidney disease. International journal of nephrology and renovascular disease, 7, 75.

Kingsley, J. D., \& Figueroa, A. (2016). Acute and training effects of resistance exercise on heart rate variability. Clinical physiology and functional imaging, 36(3), 179-187.

Kuller, L. H., Eichner, J. E., Orchard, T. J., Grandits, G. A., McCallum, L., Tracy, R. P., \& Multiple Risk Factor Intervention Trial Research Group. (1991). The relation between serum albumin levels and risk of coronary heart disease in the Multiple Risk Factor Intervention Trial. American journal of epidemiology, 134(11), 12661277.

Lee, I. M., Sesso, H. D., Oguma, Y., \& Paffenbarger Jr, R. S. (2003). Relative intensity of physical activity and risk of coronary heart disease. Circulation, 107(8), 1110-1116.

Lewis, M. J., \& McNarry, M. A. (2013). Influence of age and aerobic fitness on the multifractal characteristics of electrocardiographic RR time-series. Frontiers in physiology, 4, 100

Meyer, K., Samek, L., Schwaibold, M., Westbrook, S., Hajric, R., Lehmann, M., ... \& Roskamm, H. (1996). Physical responses to different modes of interval exercise in patients with chronic heart failure - application to exercise training. European heart journal, 17(7), 1040-1047.

Poddar, M. G., Kumar, V., \& Sharma, Y. P. (2015). Automated diagnosis of coronary artery diseased patients by heart rate variability analysis using linear and non-linear methods. Journal of medical engineering \& technology, 39(6), 331-341.

Sekine, M., Izumi, I., Yamagami, T., \& Kagamimori, S. (2001). Obesity and cardiac autonomic nerve activity in healthy children: Results of the toyama birth cohort study. Environmental health and preventive medicine, 6(3), 149.

Skrapari, I., Tentolouris, N., Perrea, D., Bakoyiannis, C., Papazafiropoulou, A., \& Katsilambros, N. (2007). Baroreflex sensitivity in obesity: relationship with cardiac autonomic nervous system activity. Obesity, 15(7), 1685-
1693.

Stein, P. K., Barzilay, J. I., Chaves, P. H., Domitrovich, P. P., \& Gottdiener, J. S. (2009). Heart rate variability and its changes over 5 years in older adults. Age and ageing, 38(2), 212-218.

Straznicky, N. E., Grima, M. T., Sari, C. I., Eikelis, N., Lambert, G. W., Nestel, P. J., ... \& Dixon, J. B. (2014). A randomized controlled trial of the effects of pioglitazone treatment on sympathetic nervous system activity and cardiovascular function in obese subjects with metabolic syndrome. The Journal of Clinical Endocrinology \& Metabolism, 99(9), E1701-E1707.

Suzic Lazic, J., Dekleva, M., Soldatovic, I., Leischik, R., Suzic, S., Radovanovic, D., ... \& Mazic, S. (2017). Heart rate recovery in elite athletes: the impact of age and exercise capacity. Clinical physiology and functional imaging, $37(2), 117-123$.

Torres Luque, G., García-Martos, M., Villaverde Gutiérrez, C., \& Garatachea Vallejo, N. (2015). Papel del ejercicio físico en la prevención y tratamiento de la obesidad en adultos (The role of physical exercise in prevention and treatment of obesity in adults). Retos, 0(18), 47-51.

Vanderlei, L. C. M., Pastre, C. M., Freitas Júnior, I. F., \& Godoy, M. F. D. (2010). Índices geométricos de variabilidade da frequência cardíaca em crianças obesas e eutróficas. Arq Bras Card, 95(1), 35-40.

Windham, B. G., Fumagalli, S., Ble, A., Sollers, J. J., Thayer, J. F., Najjar, S. S., ... \& Ferrucci, L. (2012). The relationship between heart rate variability and adiposity differs for central and overall adiposity. Journal of obesity, 2012.

Wisløff, U., Nilsen, T. I., Drøyvold, W. B., Mørkved, S., Slørdahl, S. A., \& Vatten, L. J. (2006). A single weekly bout of exercise may reduce cardiovascular mortality: how little pain for cardiac gain?'The HUNT study, Norway'. European Journal of Cardiovascular Prevention \& Rehabilitation, 13(5), 798-804.

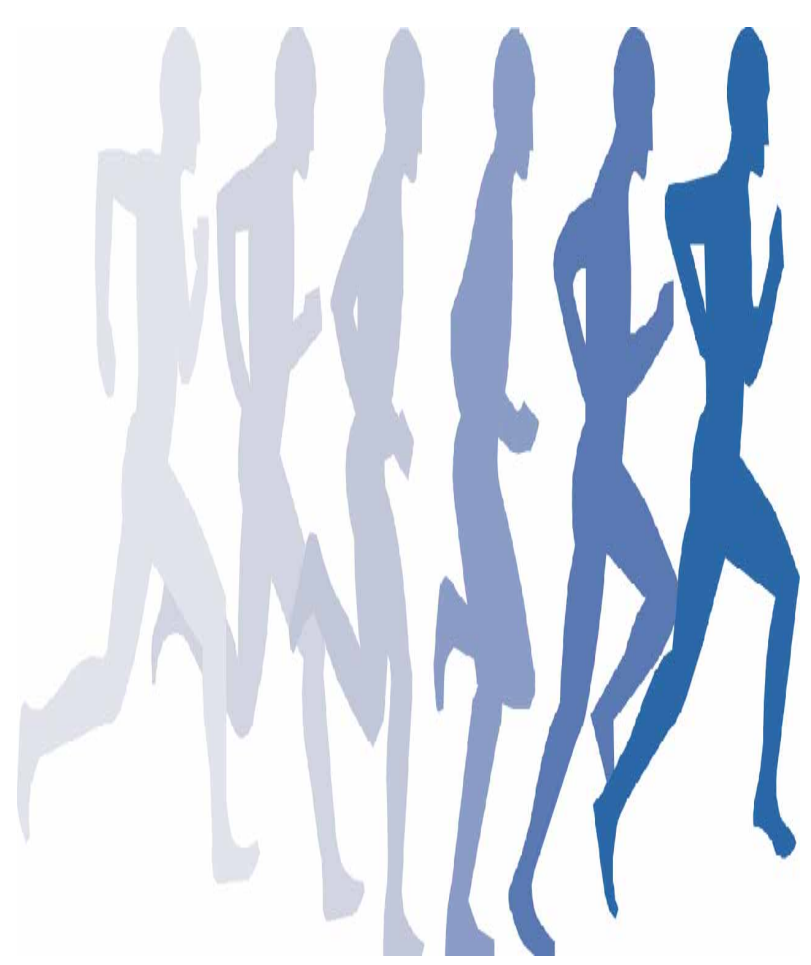

